\title{
GRIPPER FINGER DESIGN FOR SPECIAL PURPOSE APPLICATIONS
}

\author{
MIKLÓS BOLERACZKI*1, ISTVÁN GÁBOR GYURIKA ${ }^{1}$, AND DÉNES FOdOR ${ }^{1}$ \\ ${ }^{1}$ Research Centre for Engineering Sciences, University of Pannonia, Egyetem u. 10, Veszprém, 8200, \\ HUNGARY
}

\begin{abstract}
The trend towards collaborative robots is resulting in these "machine workers" working alongside humans in many workplaces. They can be used for a wide range of tasks and, most importantly, can rapidly switch between tasks. When it is no longer necessary for them to work at one location, they can simply be transferred to support another production process. With their universal gripper they can handle a wide range of tasks, however, custom accessories may need to be produced in specific areas. One such accessory could be sleeves that are mounted on the grippers to extend the use of the universal gripper. This paper aims to provide assistance on how to design these fingers.
\end{abstract}

Keywords: collaborative robot, gripper finger, 3D-printed jaw

\section{Introduction}

Robotization is a common solution in industrial environments. Despite being very expensive, it offers a relatively quick return on investment. In addition to the robot, the cost of robotization includes other service and auxiliary units such as effectors, feeders, fixtures and tooling. How is a robot gripper chosen? [1] For example, on the basis of dexterity, how much it can grip and how much it can open. Gripper fingers are applied less than small jaws which are preferred because of their flexible elements.

One of the significant advantages of collaborative robots, which are currently emerging in industry, is that they can be used to conduct multiple tasks with the need for minimal modifications. In the past, installed and enclosed industrial robots were usually purchased as well as prepared for a single purpose, typically to be operated in a production cell. Currently, collaborative robots often perform a range of tasks in collaboration with humans [2], partly because if a robot is no longer needed at one workstation, it can be used on another line at another workstation. As a result, the amount of reassembly and installation work that maintenance and line engineers are required to do is minimal.

\section{Gripper selection}

It is recommended to choose a solution for the robot effector that is best suited to such a general manufacturing environment. Although grippers are available in a variety of sizes, it is also worth considering their cost. In general, a more expensive gripper can handle multiple tasks,

\footnotetext{
*Correspondence: boleraczki.miklos@mk.uni-pannon.hu
}

while a cheaper version may be less capable of adapting to the task at hand.

The gripper is a mechanical interface between the robot and its environment [3]. Without it, the robot cannot perform its task, e.g. packing or assembling. The cited article introduces various designs of grippers. The first group of gripper fingers include several notches in the gripping surfaces, which make it possible to grip workpieces of different shapes. This is usually only suitable for workpieces of a similar size and weight. Even though they are simple to make and low-cost, the number of notches that can be cut is limited. Since the number of notches required for all workpieces must be specified, the design time may be longer. Programming the robot can also be a lengthy process because the gripper must be correctly oriented to the workpieces. Another method is to change the fingers of the gripper so that differently shaped workpieces can be handled by changing the gripper fingers. The fingers are stored on a rack and the robot knows the exact position of each finger. A reliable mechanical device to accurately clamp and unclamp the fingers is essential in this case, moreover, their replacement time should be minimized. Therefore, the gripper and robot will be able to handle a wide range of workpieces and easily adapt to other applications. This method is more flexible than the previous one, which is more application-specific.

The third technique is to replace grippers. For this purpose, complete tool changer grippers can be purchased, which are expensive but precise and allow grippers to be quickly replaced. They are used when a gripper cannot handle the differences in size, geometry and weight between workpieces. Therefore, several grip- 
pers are needed to cover a range of workpieces. The robot must be provided with precise information about each gripper and its position, which usually also requires a power supply and sensors. In general, this gripper changeover can be used for different types of grippers, e.g. mechanical, vacuum, magnetic, etc. As the changeover time increases the downtime, it should be kept to a minimum. Although modern tool changers are equipped with a number of energy transfers (air and electrical connections), they are expensive.

A fourth solution is to attach several grippers to the robot simultaneously in a revolving or linear arrangement. In the simplest case, two grippers are mounted for loading/unloading operations, which significantly speeds up the service time of the machine. The fifth option presented in this article are active and passive universal grippers. Grippers that can adapt to the workpiece, e.g. in an elastic manner, with a passive (non-actuated) degree of freedom can be considered as passive universal grippers. They are characterized by the fact that they do not provide a precise position of the workpiece in the coordinate system of the robot. They are well-suited for simple pick-and-place tasks where a high degree of precision is not required. Active universal grippers mimic the universal gripping ability of human hands. Even though such experiments have been conducted in the past, nowadays, with the addition of 3D printing, some open source projects include such active universal robotic hands. At the time of writing that article, these robotic hands were expensive and unreliable so inapplicable on an industrial scale, which is still true today. Although they are constantly being improved and better products are being developed, their real applications are in the service robotics market rather than in industrial manufacturing despite research being carried out in this field as well [4].

Zubair et al. have developed [5] an attachable core gripper for a collaborative robot that can be operated without the robot using its own power source, which is an uncommon solution in the market. Detachable robotic grippers not only work as a fully functional gripper when attached to the robot but also once detached. In terms of their physical design, 4 electromagnets are located on the side which is attached to the robot, while on the other side, 4 fingers grasp objects. The whole device is located in a 3D-printed housing. The usability of their gripper has also been tested by 12 participants. The development solution is predominantly intended to be used in a nonindustrial environment.

Long et al. designed a multifunctional gripper for grasping general objects [6] because many places, e.g. warehouses, require simple pick-and-place tasks and the level of automation is increasing due to rising costs of manpower. Underactuated grippers consist of two fingers, each of which has three degrees of freedom with a fivebar mechanism actuated by two motors. Experience has shown that the gripper, which combines two types, is versatile and reliable for the purpose of manipulating a wide range of objects.
In terms of workpieces, it is easiest to categorize the many different products into families, thereby simplifying and economizing the process required to select grippers. According to a survey of 1,000 workpieces, the most necessary requirements for a gripper are an inexpensive rugged type of jaw with a stroke of $50 \mathrm{~mm}$ and a clamping force of less than $100 \mathrm{~N}$.

\section{Promising attempts}

Current conventional robotic grippers have a number of drawbacks, namely their large volume, high weight as well as energy consumption and significant cost. A promising trend is the exploration of shape memory alloy-actuated grippers. Shape memory alloys (SMAs) can change their shape under stress [7]. The gripper presented in this research is powered by $9100 \mathrm{~mm}$-long SMA wires with a diameter of $0.4 \mathrm{~mm}$. The wires are tied together sequentially so that their displacement is added and the opening of the stroke gripper is long. Conventional grippers have servo or stepper motors and pneumatic or hydraulic actuators, rendering the gripper heavy and expensive as well as requiring a high power consumption. In contrast, SMAs as actuators are characterized by their high energy density, low energy consumption, quick response and repetitive actuation. Another special feature of the gripper presented in this article is that it has four parallel jaws on one finger. The outer two are rigid, while the inner two are flexible. At the time of writing, the gripper also has some defects, the most critical of which is that since the SMA wire is cooled naturally, the time required for the finger to close is more than 5 seconds.

Horacio Leon et al. are developing a robotic hand that consists of 5 fingers, with an extra thumb instead of a little finger [8]. The thumb is important for two reasons, namely for precision grip and power grip. The thumb is particularly critical because it increases the functionality of the hand by $60 \%$. In the article, the concept DFAM (design for additive manufacturing) was used to develop the object quickly and cheaply by building it layer by layer using a variety of materials, that is, plastic, ceramic, metal or even concrete and glass. The method is based on reducing the amount of resources, print time, weight and cost. It also brings about quality enhancement like strength and functionality. A common practice is referred to as "remixing,"- that is, a 3-dimensional design refers to other designs that are used. Eight different versions of remixing have been created, the first of which is the open InMoov robotic hand, which consists of 36 parts and 17 degrees of freedom, namely its parameters are identicalto those of the human hand. The first remix version, "Parloma Hand," has 22 degrees of freedom. The article also gives an important account of the methodology, which consists of three main parts: inspiration, ideation and implementation. By its very nature, remixing is an iterative process, which is also reflected in the methodology. Finally, a gripper was developed that has two thumbs, one 
on both the right and left side of the hand, with three fingers in between them. The hand called "Kool" is composed of 33 components and has 19 degrees of freedom, moreover, its weight, cost and printing time has been reduced compared to the original hand. Since the thumb can also grip more firmly, it is also more secure and, therefore, more functional.

Based on the aforementioned classification, the types of grippers created in the context of soft robotics can be considered as active universal grippers, which do not essentially have rigid articulated links but can be described by continuous kinematics. Developers expect these to be more effective in everyday life because they better replicate the structure of living beings, i.e. given that most of them are made of soft materials rather than rigid ones, they are more adaptable to changes in the environment. Although some connect rigid members with hinges, other studies have experimented with grippers made of a material that is truly continuously soft. An example of this is the printed soft gripper [9] presented in the article by Slesarenko et al. Their method involved inserting wires and inserts into a polymer gel in various ways to create a more controllable as well as deformable shape and even reduce the actuating force required. The basic principle of a hollow polymeric fuselage thread was used and the shape as well as internal design of the fuselage varied by sectioning it, stiffening it in certain places and weaking it in others. The resulting gripper is suitable for manipulating small objects. The recommended strategy can be applied to other types of soft actuators. Based on the presented gripper, it would be worthwhile to investigate how these grippers can be combined with conventional ones.

Nowadays, the greatest need is for a gripping system that can quickly adapt to the task. Robotic head exchange systems are available that typically consist of two parts, one to be placed on the robot and the other on the gripper, which can then be connected together. More than one gripper can be used to adapt the robot to suit different tasks. This system requires several grippers and the tool changer must be purchased as well as integrated. Another problem is the weight of the head exchanger, which is grooved according to the payload of the robot. Typically, this is undesirable for companies wishing to use collaborative robots for multiple tasks. A better solution might be to attempt to use the gripper for each significantly different task by changing the finger itself. These fingers can be precisely fitted to the gripper, moreover, are relatively simple to manufacture and replace. Additive manufacturing can be used to reduce production time and costs.

\section{Methods to design fingers of the gripper}

According to the design methodologies, the easiest solution would be to use a given template. By following a series of well-established steps, a finger can be obtained that is suitable for the current gripper and task. By following these steps, the design time is also significantly reduced because it is unnecessary to intuitively guess a solution, which can take an uncertain amount of time. The easiest way to proceed is to create a spreadsheet or flowchart in which the following questions can be answered to achieve the required outcome:

1. What type of gripper is used?

2. What product is to be gripped?

3. How accurate is our robot?

4. What material are the gripper fingers composed of?

The proposed methodology shown in Fig. 1 builds on the main elements outlined above with a more detailed description as follows. Firstly, which component (or families of components) are to be manipulated needs to be determined. Its size, weight, geometry, surface quality and material need to be taken into consideration. Furthermore, the robot gripper must be analyzed to determine the type and size of its opening as well as how the gripper fingers can be connected to it. The next step is to start the design process, which requires knowledge of the manufacturing technologies available in the factory, namely the $3 \mathrm{D}$ printing machines themselves, their characteristics and the type of materials used. The design process should be carried out by bearing in mind the aspects discussed above with a focus on good printability. Finally, based on the design, the $3 \mathrm{D}$ printing of the fingers results in physical parts. The solution to the problem is then tested by mounting the gripper fingers on the robot.

Replicating the grip and timing of the fingers is sought. For this purpose, the quickest and most efficient manufacturing solution is 3D printing. Since numerous excellent review articles on 3D printing have been published, researchers are easily provided with an up-to-date overview of the technology [10]. The article presents a comparative analysis of the available technologies and materials. As various additive manufacturing machines are becoming available in more and more factories, it is important to understand the manufacturing requirements that need to be met in the design [11]. By taking into consideration the most common FDM (Fused Deposition Modeling) machines, it is recommended that the printed finger has a flat surface which can be placed on the print bed. By maintaining an angle of $45^{\circ}$ when designing upwardly extending gripper fingers, the finger can extend the gripper's field of application by increasing its maximum opening width. It can also be adapted to suit more precise gripping tasks by designing shaped fingers.

Universal grippers can also meet specific needs. Other design considerations are:

1. The fingers should consist of one or more pieces;

2. The fingers should be composed of at least one material and produced by at least one technology.

3. In 3D printing, composite materials, both continuous and short fiber-reinforced, are also commonly used [12].

The design should take into consideration both sides, one of which is the surface of the workpiece. Between the two 


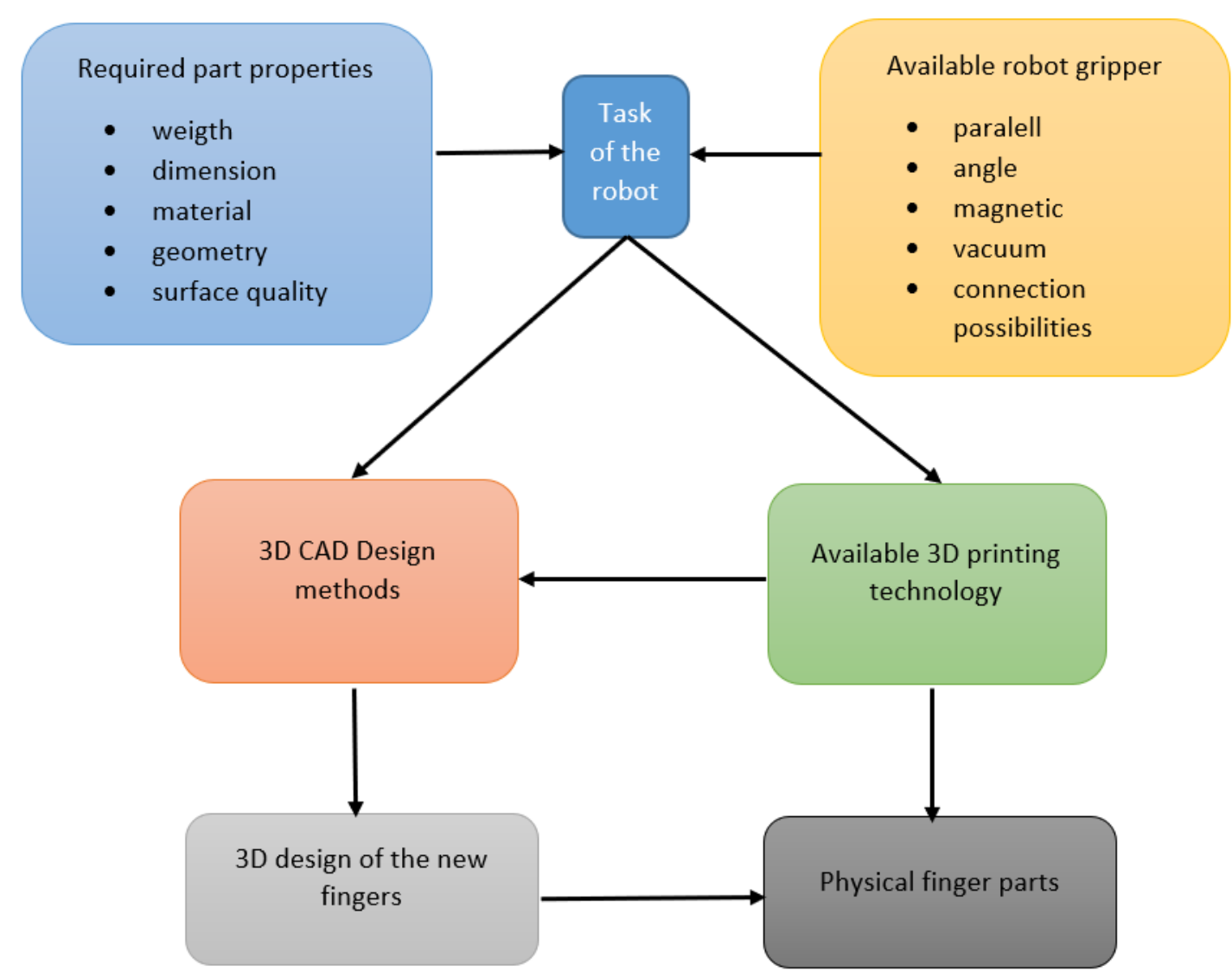

Figure 1: Methods of designing and manufacturing fingers

main components, a form that can be printed easily must be fitted. The available 3D printing processes must be assessed and the one that best suits the task selected. PLA (Polylactide), ABS (Acrylonitrile butadiene styrene) and nylon are the most common materials used by FDM machines.

\section{Example design of a finger}

Based on the aforementioned methodology, two examples are presented in Fig. 2. Firstly, in order to grip a cylindrical workpiece, it is necessary to extend the opening width. Besides, the finger has a prismatic shape, which renders the gripping concentrical. In this case, the limitation of this printing technology, namely the $45^{\circ}$ overhang, must be taken into consideration. In the second image, a small PCB (printed circuit board) must be moved by fingers, which include notches to guide their orientation against the short gripping surface. In this finger design, the $45^{\circ}$ overhang is not important because of its small dimensions. The gripper is an RG2-type universal gripper with an electric motor. 3D printing can be used to produce a rapid prototype for the purpose of experiments, which is advantageous due to its rapid nature and as the fingers can be produced very cheaply.

\section{Summary and future work}

Choosing a suitable gripper is important in terms of its application and once selected, it must be implemented as effectively as possible. The present paper aimed to provide guidance on how this can be achieved by extending its range of uses with custom-designed fingers. In the future, it is expected that composite $3 \mathrm{D}$ printers will become more widespread in the industrial environment, fur-
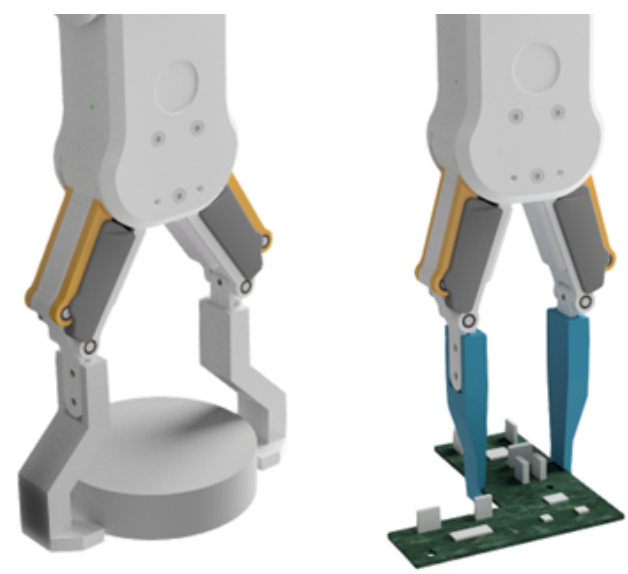

Figure 2: Cylindrically shaped workpiece and PCBgripping fingers 
ther expanding the application areas covered by 3D printing. A similarly exciting topic would be the integration of mobile robots into the manufacturing environment, for which additive manufacturing could also be used.

\section{Acknowledgment}

This work was supported by the TKP2020-NKA-10 project financed under the 2020-4.1.1-TKP2020 Thematic Excellence Programme by the National Research, Development and Innovation Fund of Hungary.

\section{REFERENCES}

[1] Pham, D. T.; Yeo, S. H.: A knowledge-based system, for robot gripper selection: Criteria for choosing grippers and surfaces for gripping. Int. J. Mach. Tools Manuf. 1988, 28(4), 301-313 DOI: 10.1016/08906955(88)90045-4

[2] Azima, M.S.; Lobov, A.; Pastukhov, A.: Methodology for implementing universal gripping solution for robot application. Proc. Eston. Acad. Sci. 2019, 68(4), 413-420 DOI: 10.3176/proc.2019.4.11

[3] Pham, D. T.; Yeo, S. H.: Strategies for gripper design and selection in robotic assembly. Int. J. Prod. Res. 1991, 29(2), 303-316 DOI: 10.1080/00207549108930072

[4] Staretu, I.: Robotic Arms with Anthropomorphic Grippers Robotic Technological Processes. Proceedings, 2021, 63(1), 77 DOI: 10.3390/proceedings2020063077

[5] Iqbal, Z.; Pozzi, M.; Prattichizzo, D.; Salvietti, G.: Detachable Robotic Grippers for Human-Robot Collaboration. Front. Robot. AI, 2021, 8, 644532 DOI: $10.3389 /$ frobt.2021.644532
[6] Kang, L.; Seo, J.-T.; Kim, S.-H.; Kim, W.-J.; Yi, B.J.: Design and Implementation of a Multi-Function Gripper for Grasping General Objects. Appl. Sci., 2019, 9(24), 5266 DOI: 10.3390/app9245266

[7] Lu, Y.; Xie, Z.; Wang, J.; Yue, H.; Wu, M.; Liu, Y.: A novel design of a parallel gripper actuated by a large-stroke shape memory alloy actuator. Int. J. Mech. Sci. 2019, 159, 74-80 DOI: 10.1016/j.ijmecsci.2019.05.041

[8] León, H.: Design for Additive Manufacturing of a Robotic Hand with Two Thumbs. In: González Díaz C. et al. (eds.) VIII Latin American Conference on Biomedical Engineering and XLII National Conference on Biomedical Engineering. CLAIB 2019. IFMBE Proceedings, vol 75. Springer, Cham. DOI: 10.1007/978-3-030-30648-9_133

[9] Slesarenko, V.; Engelkemier, S.; Galich, P.I.; Vladimirsky, D.; Klein, G.; Rudykh, S.: Strategies to Control Performance of 3D-Printed, CableDriven Soft Polymer Actuators: From Simple Architectures to Gripper Prototype. Polymers, 2018, 10(8), 846 DOI: 10.3390/polym10080846

[10] Krajangsawasdi, N.; Blok, L.G.; Hamerton, I.; Longana, M.L.; Woods, B.K.S.; Ivanov, D.S.: Fused Deposition Modelling of Fibre Reinforced Polymer Composites: A Parametric Review. J. Compos. Sci., 2021, 5(1), 29 DOI: 10.3390/jcs5010029

[11] Diegel, O.; Nordin, A.; Motte, D.: A Practical Guide to Design for Additive Manufacturing, Springer, 2020 DOI: 10.1007/978-981-13-8281-9

[12] Wang, K.; Li, S.; Rao, Y.; Wu, Y.; Peng, Y.; Yao, S.; Zhang, H.; Ahzi, S.: Flexure Behaviors of ABSBased Composites Containing Carbon and Kevlar Fibers by Material Extrusion 3D Printing. Polymers, 2019, 11(11), 1878 DOI: 10.3390/polym11111878 